\title{
Noninvasive Electrocardiographic Imaging of Scar-Related Ventricular Tachycardia: Association With Magnetic Resonance Scar Imaging
}

\author{
Omar A Gharbia ${ }^{1}$, Susumu $\mathrm{Tao}^{2}$, Albert C Lardo $^{2}$, Henry Halperin ${ }^{2}$, Linwei Wang ${ }^{1}$ \\ ${ }^{1}$ Rochester Institute of Technology, Rochester, NY, USA \\ ${ }^{2}$ Johns Hopkins Hospital, Baltimore, MD, USA
}

\begin{abstract}
A common setting for scar-related ventricular tachycardia is a reentry circuit formed by narrow channels of surviving tissue inside the myocardial scar. It is challenging to identify the critical components of these circuits using invasive catheter mapping due to its inability to map the vast majority of unstable VTs. While electrocardiographic imaging (ECGi) provides a promising noninvasive solution for rapid mapping of unstable VTs, its validation in the setting of scar-related VT remains challeging. In this paper, we report our initial results in the effort to integrate ECGi results with late gadolinium enhanced cardiac magnetic resonance imaging ( $L G E-c M R)$ of scar. We report quantitative association between ECGi features and CMR scar data, as well as qualitative relation between ECGireconstructed VT circuits and myocardial scar and critical channels identified from LGE-CMR data.
\end{abstract}

\section{Introduction}

Scar-related ventricular tachycardia is a major cause of sudden cardiac death, with an estimated 300000 cases in USA alone [1]. Reentry is the most common mechanism of Scar-related ventricular tachycardia [2], which involves a "short circuit" formed by narrow channels of slow-conducting surviving tissues $\mathrm{z}$ inside the myocardial scar. Accurate identification of these channels and their exit sites from the scar can allow precise targeting of VT through radiofrequency ablation [3]. This however is a challenging task because the current state-of-the-art catheter mapping - can only map up to $10 \%$ VT circuits that are stable in time and involve challenges such as invasive catheter maneuvers and limited sampling density [4].

Electricardiographic imaging (ECGi) is a non-invasive approach to reconstructing electrical signals on the heart by combining high-density surface ECGs and geometries of the heart and torso [5]. It has the potential to provide a noninvasive adjunct to catheter mapping by allowing rapid mapping of all inducible stable or unstable VTs, and by allowing electrical mapping of scar substrates in a completely noninvasive manner before the procedure [6].

The validation of ECGi in scar-related VT, however, remains challenging. There have been several studies that examined ECGi of scar-related VT on a small group of human subjects [7]. Most of these studies, however, are limited to qualitative validation of the ECGi-identified VT exit sites and reentrant patterns. This is largely due to the lack of gold standard data regarding the VT circuit and the culprit conducting channels. Limited quantitative validation studies include two animal case studies that involves a torso-tank set up [1], and a recent human case study where ECGi solutions were compared to both late Gadolinium enhanced cardiac magnetic resonance (LGE-CMR) data and cathether mapping data [8].

In this paper, we evaluate ECGi solutions with respect to high-resolution LGE-CMR imaging of myocardial scar. We first consider the association between sinus-rhythm ECGi-computed electrical features and LGE-CMR identified scar tissue. We then analyze the morphology and exit sites of ECGi-computed VT circuits with respect to the morphology and critical channels identified from LGECMR images. We demonstrate the electroanatomical associations between ECGi and CMR data, and argue that integrated MRI-ECGi analysis may provide important insights into the understanding of a VT circuit.

\section{Methods}

\subsection{Experimental procedure}

The animal model protocol used in this study - as illustrated in Fig.1, was approved by the university Animal Care and Use Committee.

2.1.1. Animal model creation: Eight swine models (35-45 kg) underwent creation of myocardial infarction (MI) by inserting a guiding catheter into the left coronary artery and occluded left anterior descending coronary artery using an angioplasty balloon [9]. 


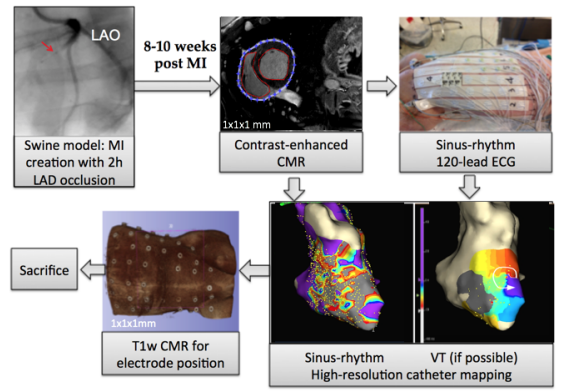

Figure 1. Summary of animal-model study procedure.

2.1.2. LGE-CMR for scar imaging: LGE-CMR was performed 8-10 weeks after the creation of MI using a 3-T scanner (Prisma, Siemens Healthcare). LGE-CMR imaging was performed 20-30 minutes after injection of Gadopentetate dimeglumine $0.20 \mathrm{mmol} / \mathrm{kg}$ (Magnevist, Bayer, Leverkusen) using a free-breathing navigator-gated three-dimensional inversion recovery $\mathrm{T} 1 \mathrm{w}$ sequence. Typical imaging parameters were $\mathrm{TI}=400 \mathrm{~ms}$, flip angle $=25^{\circ}, \mathrm{TR} / \mathrm{TE}=5.4 / 2.7 \mathrm{~ms}$, reconstructed pixel size $1.1 \times 1.1 \times 1.1 \mathrm{~mm}$ with interpolation in the slice direction, 12 segments per imaging window, GRAPPA acceleration factor $(\mathrm{R})=2, \mathrm{FOV}=300 \times 220 \mathrm{~mm}$, matrix $272 \times 200$, bandwidth $=200 \mathrm{~Hz} /$ Pixel, scan time $=15-20 \mathrm{~min}$.

2.1.3. Electroanatomical mapping with simultaneous 120-lead ECG: One week after the LGE-CMR, an electrophysiological study was performed. Prior to the electrophysiological study, 18 strips of 120 ECG electrodes were placed on the swine torso. In heparinized animals, endocardial mapping of the left ventricle was performed during sinus rhythm via a retrograde approach using a duodecapolar (20-electrode) catheter (AfocusII, 1-2.5-1 mm interelectrode spacing, 1-mm electrode size, St. Jude Medical, Minnetonka, MN) with the NavX mapping system. To induce VT, programmed ventricular stimulation was performed from 2 right ventricular sites with up to 3 extrastimuli decremented to ventricular refractoriness.

Body-surface ECG were recorded using the standard Dalhousie mapping protocol [5]. Later, to record surface ECG during induced VT, 10 surface electrodes had to be removed to accommodate the placement of NavX pads. The rest of the electrodes remained attached to the body.

2.1.4. MRI anatomical imaging: Stacks of $2 D$ MRI images were performed to identify the ECG electrodes. Imaging parameters for a T1-weighted gradient echo sequence with fat suppression were: reconstructed pixel size $1.1 \times 1.1 \times 1.1 \mathrm{~mm}, \mathrm{FOV}=400 \times 400 \mathrm{~mm}$, matrix $384 \times 307$, bandwidth $=620 \mathrm{~Hz} /$ Pixel, breath hold for 20 seconds [8].

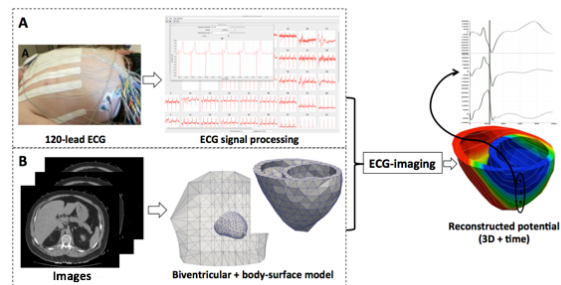

Figure 2. ECGi pipleline. A: ECG pre-processing. B: Construction of patient-specific geometrical models. From these data, ECG-imaging generates eipcardial and endocardial electrocardiograms.

\subsection{ECGi}

ECGi pipeline used in this study is illustrated in the figure 2, with three main components: 1) 120-lead bodysurface , 2) the forward model constructed from subjectspecific heart-torso models,3) and the inverse method.

2.2.1. Anatomical model and forward matrix: Geometries of the heart and torso were obtained using MR images of the heart and torso. From short-axis CMR images, contours of the epicardial and endocardial surfaces were manually extracted and used to construct a biventricular surface mesh. An isotropic surface model of the torso was constructed from the surface electrode locations manually extracted from thorax MRI images. Given the heart and torso models, the open-source SCIRun toolkit [10] was used to construct the forward model that represents the relation between cardiac activities and body potentials.

2.2.2. ECG processing: A custom research software (ECGViewer, Rochester Institute of Technology, Rochester, NY) was used to pre-process 120-lead ECG signals. Bad leads were manually discarded. Noises were removed by wavelet filtering, and baseline correction was performed by fitting and subtracting from the signal a polynomial approximation of the waveform [6].

2.2.3. Inverse method: To reconstruct unipolar electrograms on the epicardial and endocardial surfaces from ECG, the second-order Tikhonov regularization was used:

$$
\hat{s}=\operatorname{argmax}\left\{\|\phi-\mathbf{H s}\|_{\mathbf{2}}^{\mathbf{2}}+\lambda\|\mathbf{L s}\|_{\mathbf{2}}^{\mathbf{2}}\right\}
$$

where the unknown potential on the heart is presented by $s, \phi$ is the body surface potential, and $\mathrm{H}$ is the forward matrix constructed from the heart and torso geometry. $\mathrm{L}$ is the Laplacian matrix of the ventricular surface.

From electrograms reconstructed during sinus rhythm, two features were extracted: the voltage was calculated as the difference between the highest and lowest deflections of each signal (QRS component), and the duration of QRS complex was manually measured. On electrograms reconstructed during induced VT, phase mapping was performed 


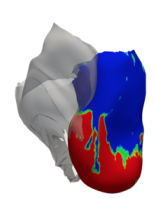

A : $10 \%$ layer of MRI

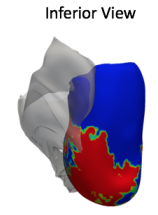

B : $60 \%$ layer of MR

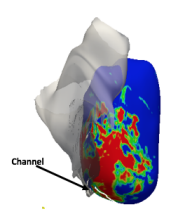

C: $90 \%$ layer of MRI
Figure 3. CMR scar delineation obtained from ADAS-VT at $10 \%, 60 \%$, and $90 \%$ of the ventricular wall.

to visually track the spatiotemporal pattern of VT [6].

\section{Results}

\subsection{Analysis of reference data}

High-resolution CMR images were analyzed using the ADAS-VT software (Galgo Medical, Barcelona, Spain). Detailed scar delineation, including scar core and gray zone, was obtained at $10 \%-90 \%$ layer of the ventricular wall at a $10 \%$ interval. In addition, potential channels were also identified at each layer, as illustrated in Fig. 3.

To corroborate the identified channels within CMR, detail analyses of catheter mapping data were also performed. This included identification of local abnormal ventricular activities [11] during sinus rhythm.

\subsection{Association between ECGi features and CMR scar data}

Pooling all sinus-rhythm results from six animals, we associated each of the two ECGi features - voltage and duration - to the three CMR-identified tissue groups (healthy, scar core, and gray zone) using a spearman's correlation test. Both features were positively associated with CMR tissue types $(\mathrm{p}<1 \mathrm{e}-10)$. As shown in Fig. 4, on the epicardium, ECGi voltage in scar core (1626.7 \pm 707.8 $\mathrm{mV})$ and gray zone $(1662.5 \pm 703.7 \mathrm{mV})$ was significantly lower than that in the healthy myocardium $(1973.6 \pm 797.2$

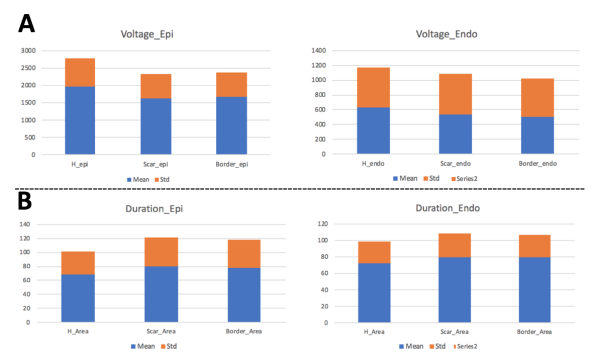

Figure 4. ECGI features. A: Epicardial and endocardial ECGi voltage in CMR healthy, scar core, and border zones. B: Epicardial and Endocardial duration in CMR healthy, scar core, and gray zones.

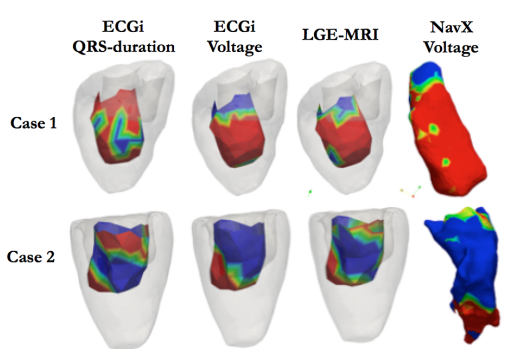

Figure 5. Visual comparison of scar maps obtained by LGE-CMR, in-vivo catheter voltage mapping, and each of the ECGi features.

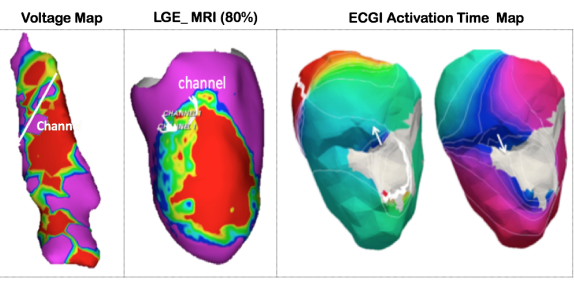

Figure 6. Critical channels as identified by CMR and catheter mapping analysis (left two columns), versus locations of the earliest activation (exit, column three) and the latest activation (entrance, column four) as suggested by ECGi reconstructed VT circuit. CMR scar is superimposed with ECGi circuit in the gray patch).

$\mathrm{mV})$. This observation held for the endocardial results, although the endocardial voltage was in general lower than that of the epicardium (healthy: $626.8 \pm 538.2 \mathrm{mV}$; scar core: $536.7 \pm 547.7 \mathrm{mV}$; gray zone: $509.2 \pm 513.8 \mathrm{mV}$ ).

In comparison, on the epicardium, ECGi-computed QRS duration was shorter in the healthy myocardium (68.4 $\pm 32.8 \mathrm{~ms})$ in comparison to that of the scar core $(80.1 \pm$ $41.2 \mathrm{~ms})$ and the gray zone $(78.2 \pm 40.2 \mathrm{~ms})$. This observation again held for the endocardium, where the ECGicomputed QRS duration was $72.1 \pm 26.5 \mathrm{~m}$ in the healthy myocardium in comparison to $79.1 \pm 29.0 \mathrm{~ms}$ and $79.4 \pm$ 27.0, respectively, for the scar core and gray zone.

For visual comparison, Fig. 5 shows endocardial examples from two animals where CMR scar maps and invivo bipolar voltage maps of scar were compared to two ECGi feature maps: ECGi voltage maps and ECGi duration maps, both thresholded to detect the region of scar. As shown, ECGi features maps, especially the voltage maps, were qualitatively consistently with CMR scar maps in the location and extent of the scar tissue.

\subsection{VT circuits with respect to CMR- delineated scar and critical channels}

Preliminary analyses were carried out to visually inspect the morphology of the ECGi-reconstructed VT circuits with respect to CMR scar and especially the anatomically identified critical channels. 


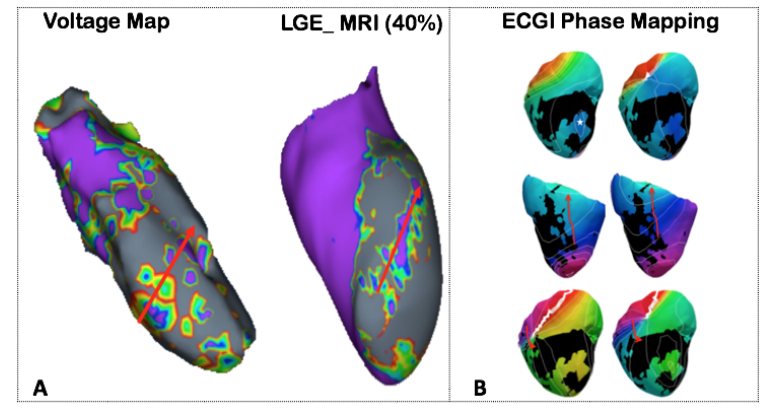

Figure 7. Critical channels. A: Channels were identified by catheter mapping and CMR. B: Phase mapping sequences of ECGi reconstructed VT circuits on the same case shown in panel A.

Fig. 6 provides an example where, in a case with anteroseptal myocardial scar, a critical channel was identified by the ADAS-VT software at the basal region of the scar near the epicardium of the heart. This channel was supported by relatively higher voltage compared to the lowvoltage scar in bipolar catheter maps. ECGi-reconstructed VT circuits exhibited an exit from the scar at the anterospetal region. After the exit, the circuit encountered an evident conduction block exactly at the region of the CMR scar (gray patch in Fig. 6). Most interestingly, the latest activation again was basal to the anteroseptal scar, in a location next to the exit. This supports the anatomical channel as identified by the CMR scar.

Fig. 7A provides another example where an anteroseptal channel was identified in the CMR at the mid- to endocardial layer, again supported by catheter voltage data. Fig. 7B shows snapshots from the phase mapping sequence of the ECGi-reconstructed VT circuits. As shown, an epicardial breakthrough was observed at the anteroseptal region of the heart near the border of the CMR scar. This circuit then exhibited a re-entrance to the anteroseptal scar, at a location basal to the exit. This pattern again indicates a consistency to the anatomical channel as identified from the CMR data. Additionally, a conduction block at the CMR scar was also observed in this ECGi-reconstructed circuit.

\section{Conclusions}

Through detailed animal model studies, this paper quantitatively demonstrated the assocation between ECGicomputed electrogram features with CMR scar delineation. Initial joint ECGi-CMR analysis also showed that ECGi-reconstructed VT circuits exhibit a morphology, especially in terms of the site of exit, entrance, and conduction delay, that is consistent with myocardial scar and critical channels as identified from CMR data.

\section{Acknowledgements}

This work was supported by the National Institute of Heart, Lung, and Blood of the National Institutes of Health under Award R21H1125998, and the National Science Foundation ACI-1350374.

\section{References}

[1] Burnes, et al. A noninvasive imaging modality for cardiac arrhythmias. Circulation 2000;102(17):2152-2158.

[2] Klug, et al. Body surface potential distributions during idiopathic ventricular tachycardia. Circulation 1995; 91(7):2002-2009.

[3] Janse, et al. Electrophysiological mechanisms of ventricular arrhythmias resulting from myocardial ischemia and infarction. Physiological reviews 1989;69(4):1049-1169.

[4] Wissner, et al. Catheter ablation of ventricular tachycardia in ischaemic and non-ischaemic cardiomyopathy: where are we today? a clinical review. European heart journal 2012;33(12):1440-1450.

[5] Hubley-Kozey, et al. Spatial features in body-surface potential maps can identify patients with a history of sustained ventricular tachycardia. Circulation 1995;92(7):18251838.

[6] Wang L, et al. Non-invasive epicardial and endocardial electrocardiographic imaging for scar-related ventricular tachycardia. EP Europace 2018; euy082.

[7] Wang, et al. Noninvasive electroanatomic mapping of human ventricular arrhythmias with electrocardiographic imaging. Science translational medicine 2011; 3(98):98ra84-98ra84.

[8] Gharbia OA, et al. Electrical and anatomical imaging of arrhythmogenic substrates for scar-related ventricular tachycardia. 2017 Computing in Cardiology CinC 2017;1-4.

[9] Ashikaga, et al. Magnetic resonance-based anatomical analysis of scar-related ventricular tachycardia: implications for catheter ablation. Circulation research 2007; 101(9):939-947.

[10] Burton, et al. A toolkit for forward/inverse problems in electrocardiography within the scirun problem solving environment. In Engineering in Medicine and Biology Society, EMBC, 2011 Annual International Conference of the IEEE. IEEE, 2011; 267-270.

[11] Jaïs, et al. Elimination of local abnormal ventricular activities: a new end point for substrate modification in patients with scar-related ventricular tachycardia. Circulation 2012; 125(18):2184-2196.

Address for correspondence:

Name: Omar Gharbia

Full postal address: 147 West Squire Dr., Rochester, NY, 14623

E-mail address: oag1929@ rit.edu 